\title{
Lorentz Violation in Torsional Antenna
}

\author{
Fabio Cardone ${ }^{1,2} \cdot$ Gianni Albertini $^{3}$ (D) . Domenico Bassani ${ }^{4}$
}

Accepted: 2 October 2020 / Published online: 12 October 2020

(c) The Author(s) 2020

\begin{abstract}
A torsional-antenna and a log-periodic antenna are used as a source and an analyzer, respectively, to investigate the possible anomalies of an electro-magnetic field. An unexpected isotropic signal has been detected using those torsion angles, which correspond to a breakdown of the Local Lorentz Invariance, which was found in the past. This coincidence is interpreted as the recovery of a lost symmetry by torqueing the antenna, thus putting in evidence that this Lorentz violation is of angular nature. Introducing a new physical dimension—not only a mathematical dimension as a way to rearrange some equations-is here proposed as a general rule to recover the lost symmetry.
\end{abstract}

Keywords Local Lorentz Invariance breakdown · Torsional antenna $\cdot$ Minkowsky spacetime $\cdot$ Metrics of interactions $\cdot$ Symmetry

\section{Introduction}

In recent years, a patent was accepted (Patent 2014) concerning a bipolar antenna having two loops torqued by a fixed angle. This antenna has a very large band of emission and a good efficiency in transmission. They are higher than in the not torqued case, thus making it particularly suitable in radio communications by satellite in asynchronous orbits.

In order to investigate the effects of the torsion angle, a systematic study was performed (Paci 2018). In particular, the reflected signal of a transmission antenna as a function of the torque angle was investigated, the not torqued case corresponding to a so-called "crossloop antenna".

Gianni Albertini

gialbe50@gmail.com

1 Istituto per lo Studio dei Materiali Nanostrutturati (ISMN-CNR), Via dei Taurini, 00185 Roma, Italy

2 GNFM, Istituto Nazionale di Alta Matematica "F. Severi”, Città Universitaria, P.le A. Moro 2, 00185 Roma, Italy

3 Dip. SIMAU, Università Politecnica delle Marche (UNIVPM), Via Brecce Bianche, 60131 Ancona, Italy

4 SIDOM S.A.S, Via Volta 34, 12010 Cervasca, CN, Italy 
In the following, we present and discuss the results obtained when a log-periodic antenna is used to study the emission of this torqued antenna. These measurements have been performed in an anechoic edge, in order to reduce the external noise.

Examples of antennas based on torsion around an axis are the Helica Filar boards (QHF) (Kilgus 1975), suitable for receiving the transmissions of the NOAA sat polar satellites and WX sat on the frequency of $137 \mathrm{MHz}$. These antennas were designed at NASA and are used in any area where a good sensitivity and an appropriate directionality are pursued. They consist of square loops twisted in a spiraling shape to form an apparent torsion angle of $180^{\circ}$; the presence of a balun and their stiffness make them different from the one we are considering and not so simple to put in evidence the effects of twisting the two loops. More recently, the influence of surface twists on the characteristics of an antenna, to be placed on the nacelle of military helicopters, was studied (Kilgore et al. 2016; Karthikeya et al. 2016).

The torsional-antenna we studied is a quadrupole, made of two concentric circular dipoles arranged on orthogonal planes. The variable torsion angle is measured with respect to this condition, in which the dipoles are on orthogonal planes. In the torqued case, we studied, the envelope is an ellipsoid of rotation. On the contrary, in the twisted case the envelope is a cylinder (or a cone), this case corresponding to helical (conical) antennas which is not matter of our study.

In this experiment, the working hypothesis was that, if any anomalous behaviour was observed, it could be put in correlation with the anomalous behaviour observed in the case of electromagnetic interaction when a resistance/capacitance circuit inside a static magnetic field was studied, at different directions (Cardone and Mignani 1999, 2000, 2004; Bartocci et al. 2001).

In that case, an unexpected voltage was observed both in the horizontal plane and in the vertical plane perpendicular to the North direction of the terrestrial magnetic field. The magnetic North direction was chosen as conventional reference direction and later on used in all the experiments and measurements which tested the Lorentz violation (Cardone and Mignani 2007; Cardone et al. 2015, 2017; Cardone 2015, 2016, 2018).

The wider Lorentz violation occurred in the vertical plane at $5 \pi / 4 \mathrm{rad}$ (Cardone and Mignani 1999, 2000; Bartocci et al. 2001) (reported in the following Fig. 4).

\section{The Antenna}

The investigated antenna is made of two vertical loops, mutually perpendicular, having almost circular shape. In fact, each loop is made of two semi-circular branches with $16 \mathrm{~cm}$ radius. Each semi-circular branch is kept at $15 \mathrm{~mm}$ apart from the central vertical axis by using two PVC cubes, one on the top and one in the bottom part. The two branches of each loop are made of harmonic steel, see Table 1, having circular section with $0.5 \mathrm{~mm}$ radius, and are closed on the top through a horizontal conducting connection of $30 \mathrm{~mm}$ which crosses the PVC upper cube. These two perpendicular connections are at a distance of $2 \mathrm{~mm}$ each other, thus avoiding the contact between the two loops. As a consequence, each loop is $100 \mathrm{~cm}$ long and $32 \mathrm{~cm}$ high. The bottom of each loop is connected to a channel of a network analyzer (Keysight Technologies N9928A) without any phase shift. About this last issue, we shall deal with in the discussion of the results. 
Table 1 Antenna wire composition

\begin{tabular}{|c|c|}
\hline \multicolumn{2}{|c|}{ Carbon steel-EN10270-1 class SH, DIN17223, ASTMA228 } \\
\hline $\mathrm{C}$ & $0.806 \%$ \\
\hline $\mathrm{Si}$ & $0.206 \%$ \\
\hline $\mathrm{Mn}$ & $0.432 \%$ \\
\hline $\mathrm{P}$ & $0.013 \%$ \\
\hline $\mathrm{S}$ & $0.004 \%$ \\
\hline $\mathrm{Cu}$ & $0.028 \%$ \\
\hline $\mathrm{E}$ & $206 \mathrm{kN} / \mathrm{mm}^{2}$ \\
\hline G & $81.5 \mathrm{kN} / \mathrm{mm}^{2}$ \\
\hline$\phi$ wire & $1 \mathrm{~mm}$ \\
\hline
\end{tabular}

Fig. 1 Vertical configuration. The torsional antenna at a torque angle $\alpha=180^{\circ}$

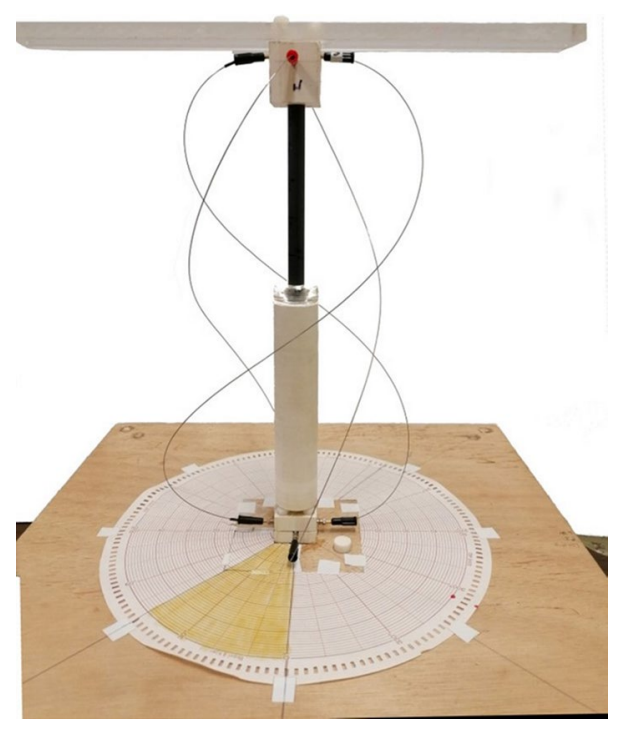

The torqued configurations were obtained by keeping fix the lower part and rotating the upper PVC cube. The torsion angle of the upper part will be indicated as the torque angle $\alpha$.

The configuration corresponding to torque angle $\alpha=180^{\circ}$ is visible in Fig. 1 .

\section{Data Collection}

The two loops of the antenna were separately power supplied by using the two output ports of an HP 8510 Vector Network Analyzer, with no phase shift, a power of $1 \mathrm{~mW}$ each on an impedance of $50 \Omega$ and a frequency of $240 \mathrm{MHz}$.

Two sets of measurements were performed on 2017 March 23rd: one with antenna axis vertical (Fig. 2) and one with the axis horizontal (Fig. 3). The time is reported in the Figs. 2 and 3.

At a distance of $220 \mathrm{~cm}$, a log-periodic antenna (Paci 2018) was placed as a receiver of the emitted signal. This log-antenna was kept fixed, while the emitting torsional-antenna 


\section{Vertical configuration}

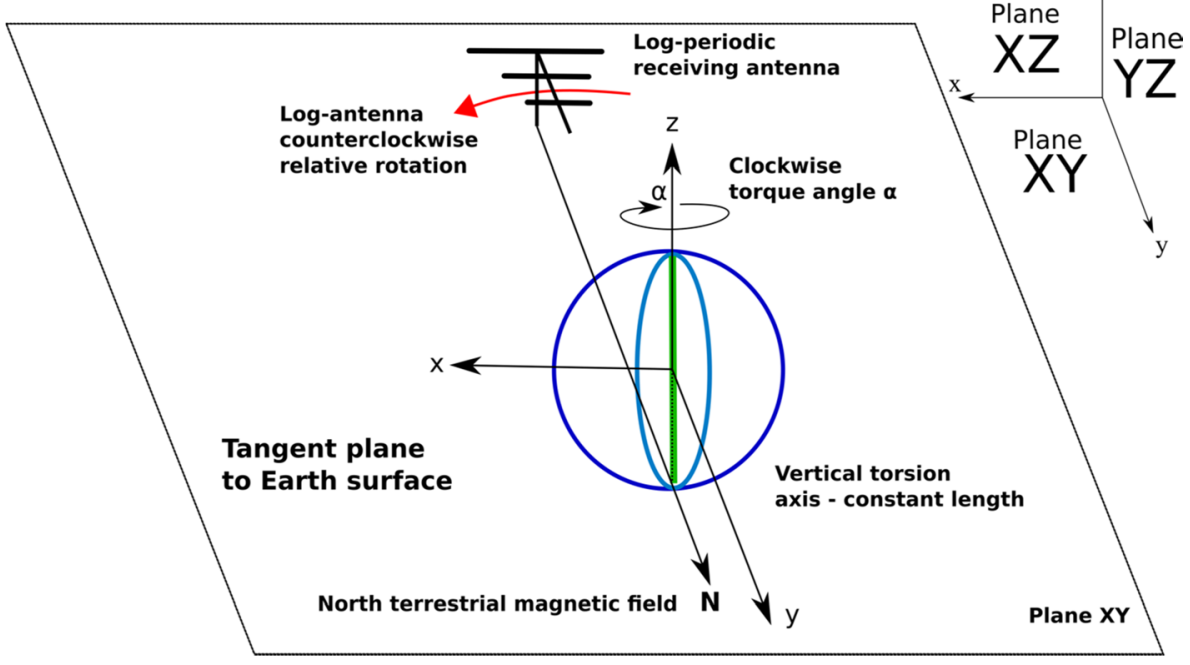

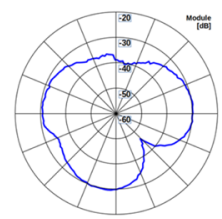

(a) $0 \mathrm{rad}$

Torque $=0^{\circ}$

h: 9:55 -9:58

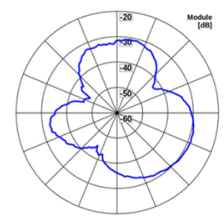

(e) $\pi \mathrm{rad}$

Torque $=180^{\circ}$

h: 10:11 - 10:14

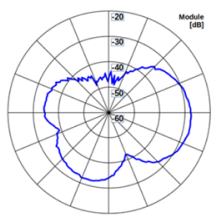

(b) $\pi / 4 \mathrm{rad}$

Torque $=45^{\circ}$

h: 9:59-10:02

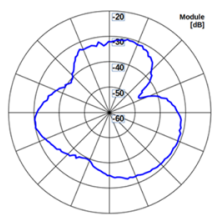

(f) $6 \pi / 5 \mathrm{rad}$

Torque $=215^{\circ}$

h: 10:15-10:18

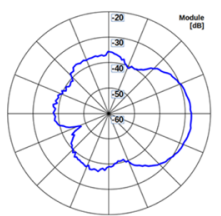

(c) $\pi / 2 \mathrm{rad}$

Torque $=90^{\circ}$

h: 10:03-10:06

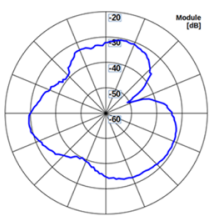

(g) $5 \pi / 4 \mathrm{rad}$

Torque $=225^{\circ}$

h: 10:19-10:22

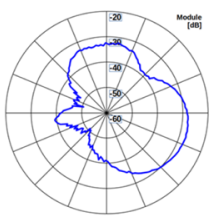

(d) $3 \pi / 4 \mathrm{rad}$

Torque $=135^{\circ}$

h: 10:07-10:10

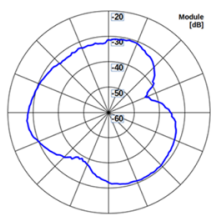

(h) $3 \pi / 2 \mathrm{rad}$

Torque $=270^{\circ}$

h: $10: 23-10: 28$

Fig. 2 Vertical configuration of the torsional-antenna: antenna torsion axis perpendicular to horizontal plane. Polar representation of intensity registered by log-periodic antenna as a function of torque angle $\alpha$, the transmitting antenna being in the vertical configuration, the units are module in the range $[-60,-20]$ $\mathrm{dB}$. Eight values of torque angle $\alpha$ are considered: $0 \mathrm{rad} \mathbf{a} \pi / 4 \mathrm{rad} \mathbf{b} \pi / 2 \mathrm{rad} \mathbf{c} 3 \pi / 4 \mathrm{rad} \mathbf{d} \pi \mathrm{rad} \mathbf{e} 6 \pi / 5 \mathrm{rad} \mathbf{f}$ $5 \pi / 4 \mathrm{rad} \mathbf{g}$ and $3 \pi / 2 \mathrm{rad} \mathbf{h}$

could rotate on a table around a vertical axis crossing the center of the antenna (rotation angle $\omega$ ), thus keeping the $\alpha$ torque-angle fixed. The total range of the rotation angle $\omega$ was $360^{\circ}$ clockwise, with 180 steps of $2^{\circ}$. At each $\omega$ step, intensity was measured during $1 \mathrm{~s}$. This way, the relative rotation of the log periodic antenna was anticlockwise. 
Horizontal configuration

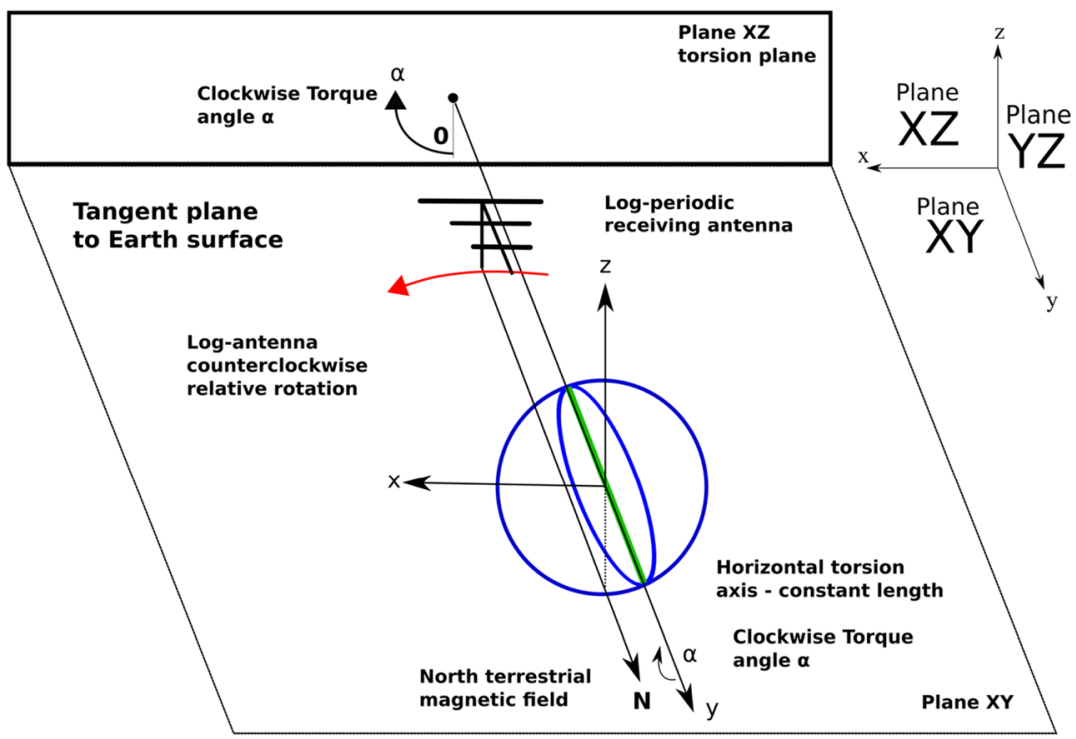

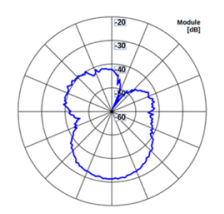

(a) $0 \mathrm{rad}$

Torque $=0^{\circ}$

h: $10: 32-10: 35$

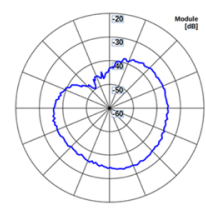

(e) $\pi \mathrm{rad}$

Torque $=180^{\circ}$

h: 10:48 - 10:51

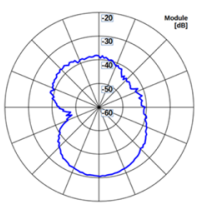

(b) $\pi / 4 \mathrm{rad}$

Torque $=45^{\circ}$

h: $10: 36-10: 39$

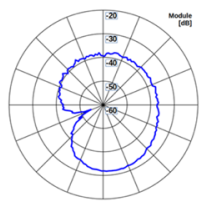

(c) $\pi / 2 \mathrm{rad}$

Torque $=90^{\circ}$

h: $10: 40-10: 43$

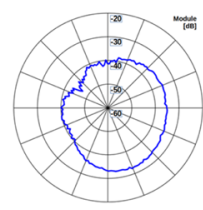

(d) $3 \pi / 4 \mathrm{rad}$

Torque $=135^{\circ}$

h: 10:44 - 10:47

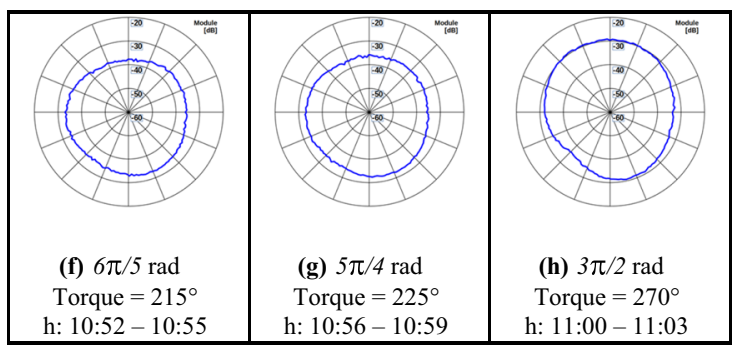

Fig. 3 Horizontal configuration of the torsional-antenna: antenna torsion axis parallel to horizontal plane and laying along the North direction. Polar representation of intensity registered by log-periodic antenna as a function of torque angle $\alpha$, the transmitting antenna being in the horizontal configuration, the units are module in the range $[-60,-20] \mathrm{dB}$. Eight values of torque angle $\alpha$ are considered: $0 \mathrm{rad} \mathbf{a} \pi / 4 \mathrm{rad} \mathbf{b}$ $\pi / 2 \mathrm{rad} \mathbf{c} 3 \pi / 4 \mathrm{rad} \mathbf{d} \pi \mathrm{rad} \mathbf{e} 6 \pi / 5 \mathrm{rad} \mathbf{f}, 5 \pi / 4 \mathrm{rad} \mathbf{g}$ and $3 \pi / 2 \mathrm{rad} \mathbf{h}$

Particular attention was paid to the orientation of the apparatus with respect to the North of the Earth magnetic field: to this aim, a cartesian coordinate system was chosen 

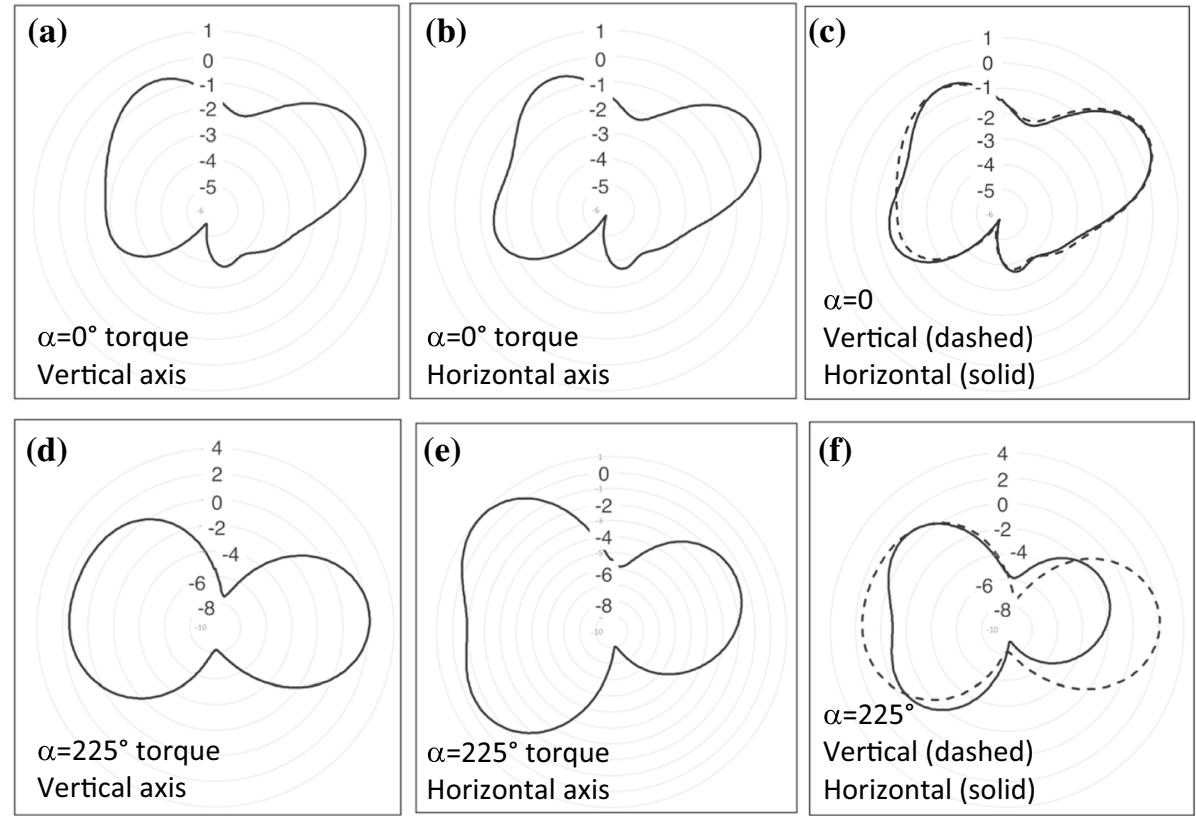

Fig. 4 Polar diagrams (in arbitrary units) by simulation at $\mathrm{a}=0^{\circ}(\mathbf{a}-\mathbf{c})$ and $\mathrm{a}=225^{\circ}(\mathbf{d}-\mathbf{f})$ in the vertical (a, d) and horizontal (b, e) torsion axis. A comparison between the horizontal (solid) and the vertical (dashed) case is reported at $\mathrm{a}=0^{\circ}(\mathbf{c})$ and $\mathrm{a}=225^{\circ}(\mathbf{f})$

having the $\mathrm{z}$ axis vertical and the $\mathrm{y}$ axis in the direction of the magnetic North $\left( \pm 2^{\circ}\right)$. The choice of such a coordinate system is devoid of any physical meaning. It is a purely conventional system, as we are going to discuss later on. With this choice, a loop was in the xz plane and one in yz plane when $\alpha=0$ and $\omega=0$ and axis of the antenna was vertical in the vertical configuration, see Fig. 2.

In the horizontal position, antenna axis was along the $y$ direction. The $\alpha=0$ and $\omega=0$ configuration corresponded to loops in the xy and zy planes (see Fig. 3: horizontal configuration).

\section{Results and Discussion}

The Fig. 2 reports the polar diagrams of the intensity registered as a function of angle $\omega$ when the antenna was in vertical configuration, the Fig. 3 refers to horizontal configuration.

The most evident effect of torqueing antenna is that the intensity distribution is almost isotropic at torqueing angles $\alpha$ larger than $\pi$ rad in the horizonal configuration, see Fig. 3 polar diagrams $\mathrm{f}, \mathrm{g}, \mathrm{h}$.

Before performing the experiment, one should have expected the most isotropic distribution at large torqueing angles in the vertical configuration, not in the horizontal. In fact, in the former case, if one considers the antenna as an assemble of many dipoles, their directional distribution is more homogeneous at high torqueing angles, thus creating an 
Fig. 5 Voltage detected in a static conductor inside e static magnetic field as a function of $\alpha$ angle formed with respect to the vertical, in a plane perpendicular to the North direction of the local magnetic field

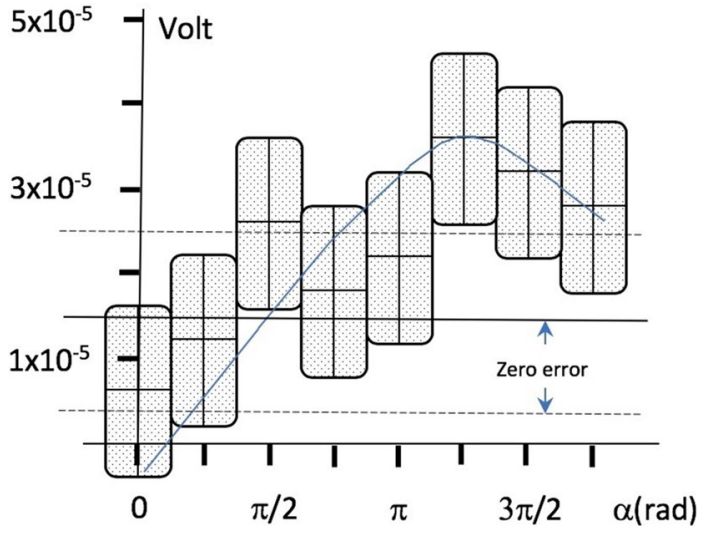

isotropic signal in a horizontal plane, which is the plane of the receiving antenna reported in the Figs. 2 and 3.

In order to check this point, a three-dimensional model of the antenna was created by using a numerical model (Healey and Mehta 2006), which has been implemented in MATLAB software. The final simulation of the electro-magnetic signal, as expected by applying standard software based on well-known classical Maxwell equations, was performed by ANSYS HFSS software. Figure 4 reports the polar diagrams obtained by simulation at $\mathrm{a}=0^{\circ}$ and $\mathrm{a}=225^{\circ}$ in the horizontal and vertical configurations.

No such effect of isotropy at high torqueing angles in the horizontal configuration was obtained (anyway no isotropic distribution has been obtained at all).

From these considerations one can deduce that the registered isotropy is not a direct consequence of the geometry, at least of the geometry we are acquainted to in a flat minkoskyan spacetime.

A possible explanation for this behaviour was looked for by considering the reported behavior of a static conductor inside a static magnetic field (Cardone and Mignani 1999, 2000, 2004; Bartocci et al. 2001).

In that case, by considering a similar conventional cartesian coordinate system with y axis along the terrestrial magnetic North and z perpendicular to the local earth surface, ${ }^{1}$ the following effects were observed:

- In the yz-plane: inside the experimental error, no voltage was detected at different directions of the conductor, as it is expected.

- In the xy-plane: inside the experimental error, no voltage was detected at different directions of the conductor, as it is expected, apart from an unexpected voltage at $\pi / 4$ (but not at 0 nor at $\pi \mathrm{rad}$ ) from the North. It was observed during the measurements from 9 to 9:59 am of the 8th of June 1998 but not between 0:00 and 0:46 pm of the same day.

- In the xz-plane, a wide variation of the voltage as a function of $\alpha$ angle from the vertical direction was detected, as depicted in Fig. 5.

\footnotetext{
1 We remind that this reference frame using the terrestrial magnetic North as $y$-axis is purely conventional and devoid of any physical meaning.
} 
The instrumental zero corresponded to $(1.5 \pm 1.0) \times 10^{-5} \mathrm{~V}$. Thus, only the measurement at $\alpha=5 \pi / 4$ is out of zero range, the error in each measurement being $\pm 1.0 \times 10^{-5} \mathrm{~V}$.

However, as the zero error is systematic (not statistical), whatever its value is, many results are different from zero. Moreover, the results are not randomly distributed, but correspond to a continuous variation with angle, as shown by the continuous line in Fig. 5. The central value of the so obtained peak is about $6 \pi / 5$, after applying correlation tests and interpolating procedure by standard polynomial regression (Cardone and Mignani 1999, 2000, 2004, 2007; Bartocci et al. 2001).

In particular, if the central value $1.5 \times 10^{-5} \mathrm{~V}$ is assumed as zero value, a wide region (at $\alpha$ larger than $\pi \mathrm{rad}$ ) corresponds to the occurrence of a voltage which must not exist, according to known behaviour of the electromagnetic interaction, i.e. without Local Lorentz Invariance (LLI) violation.

The results reported in Fig. 5 were obtained between 8:30 am and 9:30 am of the 9th of June 1998. A following measurement between 12:18 and 13:05 pm of the same day did not confirm this occurrence of an anomalous voltage. These results have been confirmed (Cardone and Mignani 2004, 2007) in the next test runs performed in the years 1999-2000: in the same period of the year and with the same geometry, a violation of the LLI of a similar amount occurred in the morning but not in the afternoon.

Both the results in the xz-plane and in the xy-plane show a dependence on the time of the experiment. Due to this fact, a dependence on a direction different than the North magnetic field was suggested. In particular, some cosmological direction, which could be related to some anomaly of the microwave background radiation and its cold spot preferred direction was proposed (Cardone et al. 2015).

At any rate, these results correspond to a breakdown of the LLI, as different results are obtained in different directions and in different times.

This anomaly occurs in directions corresponding to those torsion angles of the antenna for which the detected signal is isotropic: angles greater than $\pi$ with respect to the vertical in a plane perpendicular to the North direction. In this angular range, a breakdown of the LLI occurred in the case of the conductor inside the static magnetic field.

In the case of the conductor in static magnetic field, this breakdown was attributed to metric parameters that are energy dependent according to energy thresholds, characteristic of each interaction (Bartocci et al. 2001; Cardone and Mignani 1999, 2004 ).

Thus, outside each threshold, the space-time is not flat for all interactions at all energy values. A not-isotropic and not-symmetric space-time corresponds to a breakdown of LLI and, as a consequence, to a breakdown of the same Maxwell equations for electromagnetism.

Thus, in the case of the conductor, a deformation of space-time was detected (Cardone and Mignani 1999, 2000, 2001, 2004, 2007; Bartocci et al. 2001).

In the case of the antenna, given that a deformation exists, the torsion of the antenna counteracts this deformation and makes the energy signal isotropic.

We remark that symmetry in the polar diagrams at low values of the torque angle $\alpha$ is not expected by simulation and is not found experimentally. Maybe some people familiar with antennas are used to find a $180^{\circ}$ symmetry. This result usually is obtained by modifying the intensity in the two loops to produce it. However, this is not the case in our experiments: this symmetry is not expected and does not occur.

In other words, the symmetric polar diagrams of Fig. 3 have been obtained without using the so-called "time's balancing" or rather without using any phase shift in separate supply for each loop. 
In this strongly asymmetric case too, isotropic diagrams were obtained at high torque angles although they are not expected according to simulation.

The followed procedure to recover the symmetry is a step ahead with respect to the already followed methods. In fact, when the three-dimensional (3D) translation symmetry of the Galileo relativity was broken (by the existence of the Lorentz force), a new symmetry was found by adding the time as a fourth dimension and the 4D Minkowsky space-time was introduced. From the 3D symmetry of the Euclidean-Cartesian space (x, y and z spatial directions, whereas $\mathrm{t}$ is the Newtonian absolute time) a 4D symmetry of the pseudo-Euclidean Minkowsky space-time (x, y, z, t) was obtained.

In our case, the violation of 4D Lorentz symmetry was recovered by adding a new variable, the energy, which - in turn - is related to the angle of Lorentz violation. Thus, a 5D space-time-energy (x, y, z, t, E) is obtained (Cardone and Mignani 2007).

From the methodological point of view, the two steps are different. In fact, the transition from 3 to 4D is a mathematical way rearranging some equations to obtain in 4D the symmetry which is lost in 3D.

In our case, on the contrary, the isotropy was obtained experimentally, thus underlying a transition from 4 to $5 \mathrm{D}$ which has to be studied in detail from the mathematical point of view. Some indications on this transition can be found in ref.s 8-10.

\section{Conclusions}

A previous experiment of a static conductor, inside a static magnetic field, pointed out the evidence that violation of the LLI invariance occurred in some directions, defined by using the convention of a plane perpendicular to the north direction of the local magnetic field of the Earth.

The torsion angle $\alpha$ of the antenna exactly follows the same convention of the orientation angle $\alpha$ of the conductor inside the static magnetic field in the coil test for the Lorentz violation, as we can see in Fig. 6a, c.

The symmetric distribution of energy in the polar diagrams exactly follows the Lorentz violation as it matches the same range of angle $\alpha$, as we can see in Fig. $6 \mathrm{~b}, \mathrm{~d}$.

As a matter of fact, in the present experiment the lost isotropy is recovered by using as source of electromagnetic field a torsional-antenna torqued by the same convention (i.e. using a plane perpendicular to the north direction of the local magnetic field of the Earth), as clearly shown in Fig. 6.

Once more we recall that the use of such a cartesian reference frame with y-axis along the North direction of the Earth magnetic field is a pure convention without any physical meaning. We stress again that this conventional reference frame has been used in all experiments testing LLI violation, either for electromagnetic phenomena or nuclear ones (Cardone and Mignani 2007; Cardone et al. 2015, 2017; Cardone 2015, 2016, 2018).

Recovering the lost symmetry by using a torsional-antenna is a clear indication that the breaking of LLI is mainly of angular nature.

Therefore, the method of introducing a new physical dimension is proposed as a general rule to recover the lost symmetry. It was already used in the past by introducing the time as a fourth dimension. This rule is used here by introducing the energy as fifth dimension (Cardone and Mignani 1999; Cardone et al. 1999a,b,c). We stress that in the present case the recovery is studied experimentally while it was introduced theoretically in the past. 


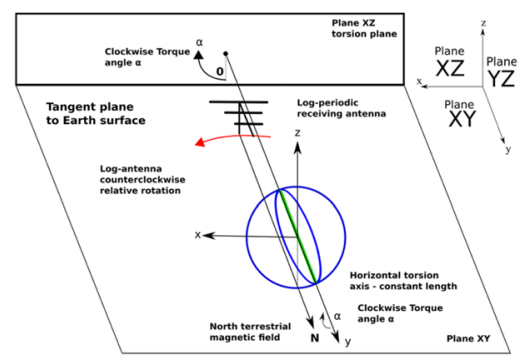

Horizontal configuration
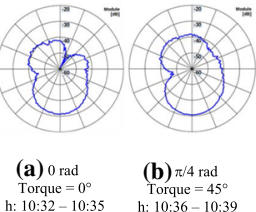

$\begin{array}{ll}\text { (c) } \pi / 2 \mathrm{rad} & \text { (d) } 3 \pi / 4 \mathrm{rad} \\ \text { Torque }=90^{\circ} & \text { Torque }=135^{\circ}\end{array}$ $\begin{array}{ll}\text { Torque }=90^{\circ} & \text { Torque }=135^{\circ} \\ \text { h: } 10: 40-10: 43 & \text { h: 10:44 }-10: 47\end{array}$

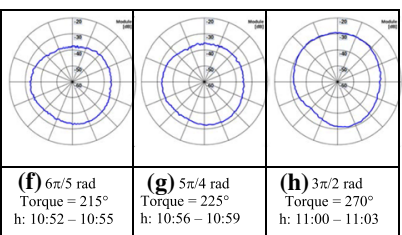

$\alpha$ torsion angle of the antenna

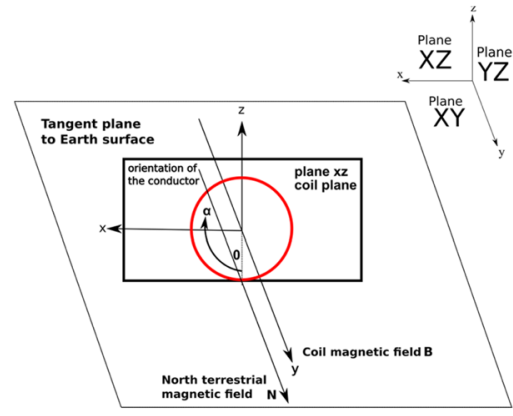

$\alpha$ orientation angle of the conductor inside the static magnetic field of the coil

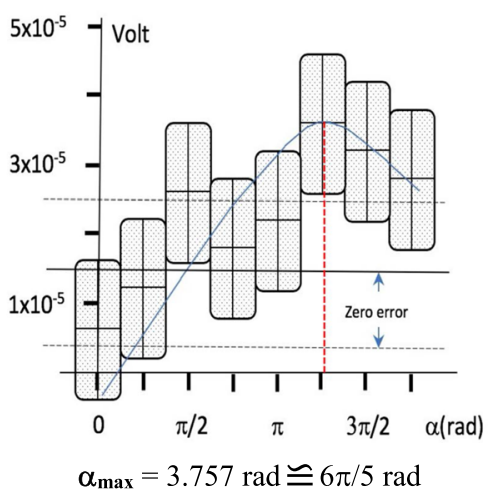

Fig. 6 a Sketch of the torsional antenna in horizontal configuration, b 8 polar diagrams of radiation on meridian plane with respect to the horizontal torque axe, $\mathbf{c}$ Sketch of the magnetic coil test for LLI violation, $\mathbf{d}$ Voltage detected in a static conductor inside a static magnetic field as a function of $\alpha$ angle formed with respect to the vertical, in a plane perpendicular to the magnetic North direction

\section{Remarks}

We noticed that the use of the energy as fifth physical coordinate allows to recover the symmetry and to solve the Lorentz violation, but the use of the fifth coordinate energy sets three problems we must face and deal with.

First, we have to deal with the energy in a separate way for each interaction, taking well in account that we measure the energy only by electromagnetic interaction (Cardone and Mignani 1999, 2004, 2007).

Therefore, this first problem can be summarized by the question on how the energy measures the energy (Cardone and Mignani 1999; Cardone et al. 1999a, b, c).

Second: which form does it assume the fifth metric parameter corresponding to the coordinate energy? This problem is also evidently connected to the question on how the energy measures energy.

Third, energy is a dynamical variable: we must examine whether the role of dynamical variable is to be extended to the other four coordinates, the three spatial ones and the time. 
This problem is also evidently connected to a geometrical representation of the interactions by metric parameters depending on the energy, intended as phenomenological variable, and pave the way to consider all the coordinates as dynamical variables.

At last, we remark that the energy has a double role in the framework of such a fivedimension metric: it is a coordinate and a phenomenological variable, on which the metric coefficients depend.

In the framework of this double role, the question on "how the energy measures the energy" finds a solution in the metric coefficient of the coordinate energy.

Therefore, we set the Ansatz that this fifth coefficient of the metric for each interaction plays the role of the gauge for the energy, and this could set a new standard to the so-called gauge field theories.

\section{Perspectives}

Among the experiments we can suggest as a step forward following the previous conclusions, the above reported measurements can be replicated in the southern hemisphere, when Earth is in the same astronomic position (i.e. between March and May).

This way, any asymmetry of the Lorentz violation can be investigated. In fact, this violation and its asymmetry were suggested to occur at a cosmological scale too (Cardone et al. 2017; Cardone 2016, 2018).

Besides, we tested the time-space asymmetry from the point of view of the space by using this torqued antenna. In order to test the asymmetry from the point of view of the time, we suggest to investigate the effects of a phase shifts between the two loops by using sinusoidal wave separate supply. Both the vertical and the horizontal configurations should be considered by using the same power supply of $240 \mathrm{MHz}, 1 \mathrm{~mW}$.

Acknowledgements It is a pleasure to thank Ph.D. Danilo Paci for sharing data from his thesis; professor Antonio Morini for allowing to use instruments of his electronic laboratory and Ph.D. Student Fabrizio Ciciulla for his valuable help in the organization and execution of measurements and data elaboration, they all being active at UNIVPM (Università Politecnica delle Marche, Ancona-Italy)

Funding Open access funding provided by Università Politecnica delle Marche within the CRUI-CARE Agreement.

\section{Compliance with Ethical Standards}

Conflict of interest No conflict of interest occurs in this work.

Open Access This article is licensed under a Creative Commons Attribution 4.0 International License, which permits use, sharing, adaptation, distribution and reproduction in any medium or format, as long as you give appropriate credit to the original author(s) and the source, provide a link to the Creative Commons licence, and indicate if changes were made. The images or other third party material in this article are included in the article's Creative Commons licence, unless indicated otherwise in a credit line to the material. If material is not included in the article's Creative Commons licence and your intended use is not permitted by statutory regulation or exceeds the permitted use, you will need to obtain permission directly from the copyright holder. To view a copy of this licence, visit http://creativecommons.org/licenses/by/4.0/. 


\section{References}

Bartocci, U., Cardone, F., \& Mignani, R. (2001). Looking for a possible breakdown of local Lorentz invariance for electromagnetic phenomena: Theory and first experimental results. Foundation of Physics Letters, 14,51 .

Cardone, F., et al. (2015). Violation of local Lorentz invariance for deformed spacetime neutron emission. The European Physical Journal Plus, 130, 55.

Cardone, F. (2018). Lorentz violation and CMRB anysotropy, In Proceedings of the 10th Symposium "Vigier”, Ancona, Italy, July 2016. Unified Field mechanics: Formulation end empyrical tests, section V. Astrophysics and Cosmology p. 369-374 (ed.,) World Scientific ISBN: 978-981-3232-03-y/04-4/05-1

Cardone, F. (2018). Lorentz violation microwave background radiation preferred directions and critical density of the universe. Journal of Advanced Physics, 7(1), 81-87.

Cardone, F., Laposta, C., \& Perconti, W. (2015). Cosmic microwave background radiation preferred directions and asymmetry in Lorentz violation. Journal of Advanced Physics, 4(2), 155-159.

Cardone, F., \& Mignani, R. (2001) "On possible experimental evidence for breakdown of Lorentz invariance". In invited lecture at international conference on relativity theory "gravitation electromagnetism and cosmology: toward a new synthesis”, Cesena, Italy 1999, (ed.,) K. Rudnicki, p. 161, Apeiron, Montreal.

Cardone, F., \& Mignani, R. (1999). Broken Lorentz invariance and metric description of interactions in a deformed Minkowski space. Foundations of Physics, 29(11), 1735.

Cardone, F., \& Mignani, R. (2000). Observation of a DC voltage induced by a steady magnetic field: A possible electromagnetic breakdown of Lorentz invariance? Physics Essays, 13(4), 645-656. https://doi. org/10.4006/1.3025454.

Cardone, F., \& Mignani, R. (2004). Energy and Geometry. Singapore: World Scientific.

Cardone, F., \& Mignani, R. (2007). Deformed Spacetime, (ed.,) Springer, Dordrecht, The Netherlands. ISBN 978-1-4020-6282-7 (HB) ISBN 978-1-4020-6283-4 (e-book).

Cardone, F., Mignani, R., \& Francaviglia, M. (1998). Energy and relativistic clock rates in five dimensions. General Relativity and Gravitation, 30(11), 1619.

Cardone, F., Mignani, R., \& Francaviglia, M. (1999a). Energy dependent phenomenogical metrics and five dimensional Einstein equations. Foundation of Physics Letters, 12, 281.

Cardone, F., Mignani, R., \& Francaviglia, M. (1999b). Five-dimensional relativity with energy as extra dimension. General Relativity and Gravitation, 21(7), 1049.

Cardone, F., Mignani, R., \& Francaviglia, M. (1999c). Energy as fifth dimension. Foundation of Physics Letters, 12, 347.

Cardone, F., Perconti, W., \& Petrucci, A. (2017). DST asymmetric emission and CMRB preferred direction. Journal of Advanced Physics, 6(2), 303-307.

Healey, T.J., \& Mehta, P.G. (2006). Straightforward computation of spatial equilibria of geometrically exact cosserat rods, world scientific series on nonlinear science series B-Modeling and computations in dynamical systems, pp. 253-269.

Karthikeya, GS., Agnihotri, N., Siddiq, SS., Mehul, KS., Thyagaraj, T. (2016). A conformal UHF antenna for cargo helicopter belly. In Antennas and Propagation (APCAP), 2016 IEEE 5th Asia-Pacific Conference pp. 285-286. IEEE.

Kilgore, I. M., Kabiri, S. A., Kane, A. W., \& Steer, M. B. (2016). The effect of chaotic vibrations on antenna characteristics. IEEE Antennas and Wireless Propagation Letters, 15, 1242-1244.

Kilgus, C. C. (1975). Shaped-conical radiation pattern performance of the backfire quadrifilar helix. IEEE Transactions on Antennas and Propagation, 23(3), 392-397.

Paci D. (2018). "Antenne di Torsione e possibili conseguenze nella Fisica Fondamentale" Ph.D.Thesis-Università Politecnica delle Marche-27th edition new series (see also: "Analysis and characterization of a twisted double loop antenna"https://arxiv.org/abs/2002.08128).

Patent n. ITBO020120598(A1)-2014-05-06.

Publisher's Note Springer Nature remains neutral with regard to jurisdictional claims in published maps and institutional affiliations.

Fabio Cardone (born in Chieti-I on October 14th, 1960) is (co)-author of more than 150 publications on Lorentz Invariance, Metrics of Interactions, Gravitation, Electromagnetism and Quantum Theory, Nuclear Physics, Particle Physics, Mathematics in Physics. His research activity is focused on the description of the 
fundamental interactions by energy dependent metrics with development of a four and five dimensions formulation of the Deformed Space Time theory from the Lorentz Invariance violation. Education and Career: 1984 Diploma at CERN (CH) and PhD in Physics at L'Aquila University (I); 1985 Master in Particle Physics, La Sapienza University, Rome -I; 1984-1989 National Institute for Nuclear Physics (INFN), RomeI; 1985 Researcher, Wisconsin University, (USA); 1985-1989 Grand Sasso National Laboratory (LNGSINFN) and Graduate Fellow of the Italian Episcopal Conference (CEI); 1989-1991 Associate Researcher at CERN (CH); 1990-1991 Visiting Assistant Professor, Syracuse University (USA).

Gianni Albertini (Ancona-I, oct.6.1950), physicist, full time professor at Università Politecnica delle Marche (UNIVPM), Ancona-I: User at main EU synchrotrons and neutron sources. Invited speaker at international congresses. Member of international scientific committees. More than 150 publications on subjects of first wall of fusion nuclear reactors, advanced materials, biomaterials (stem cell, model membranes, prosthetics), liquid crystals, computational physics and engineering simulation, strain/ stress, texture, aeronautics, automotive, food and nuclear industry; geology, nuclides and nuclear reactions. He took part at the realisation of neutron diffractometers in UK in the framework of EU project PREMIS and in France in the framework of LLB(F)-INFM(I) joint venture, Referee for international journals. Deputy Rector of the university at the assembly of EU Virtual Institute KMM-VIN and assembly of CNISM (national inter-university consortium for physical sciences of matter).

Domenico Bassani PhD (Engineer. Born: 1959, July 22 in Turin-I) $(\mathrm{Ph}+39333$ 6459510. Email domenico.bassani@gmail.com). He has participated in national and international congresses mainly concerning Nuclear Reactions in Condensed Matter. His research activity is mainly focused on subjects related to reactions in Deformed Space-Time and violation of Local Lorentz Invariance. He designed and built up the instrumentation necessary to carry out these reactions. He is co-author of publications on experimental and theoretical Physics in international journals with referees. Education: 1991-1994 PhD in Construction and Machine Design at the Department of Mechanics of the Polytechnic of Turin- I; 1990-1991 Degree in Mechanical Engineering at the Politecnico di Torino-I; 1972-1977 Scientific high school San Giuseppe College (Turin-I). 